\title{
Evolution mechanism of ultraviolet and electrical phenomena induced by protrusion discharge in GIS
}

\author{
Fei Du${ }^{1}$, Wenzhi Chang ${ }^{1}$, Jiangang $\mathrm{Bi}^{1}$, Shuai \\ Yuan $^{1}$, Xinghui Zhang ${ }^{2}$, Yanpeng Gong ${ }^{1}$
}

\begin{abstract}
To improve the sensitive and reliability of condition monitoring in gas insulated switchgear (GIS), discharge perception system applied to GIS insulators protrusion discharge based on single ultraviolet photon is built. Step method is used to inspire the discharges of different severity, based on which, the regression relationship of ultraviolet (UV) signal and electrical signal are studied, and the perception mechanism of UV signal in different severity of discharges is analyzed. The study indicates that, the development of protrusion discharge in GIS can be divided into four stages, and there is no positive correlation between the number of ultraviolet photons caused by discharge radiation and insulation deterioration level, but related to the diffusion, migration and recombination of charged particles, then UV optical method for quantitative monitoring of discharge in each stage is provided, which provide a new method for quantitative measurement of GIS insulator discharge.

K e y w or ds: GIS insulator, perception technique, single photon detection
\end{abstract}

\section{Introduction}

With the development of power grid in the direction of high voltage, large capacity and intensive, the requirements of reliability and stable operation are further improved, and gas insulated switchgear (GIS) has been widely used. By 2019, the main fault of GIS is insulation fault. In particular, flashover caused by foreign matters accounts for about $50 \%$, and the internal insulation fault of protrusion discharge accounts for about 30\%. Partial discharge (PD) is an important characterization of GIS insulation degradation. For the online GIS, the main detection methods are based on ultrasonic and ultra-high frequency (UHF) technique, as the signal propagation of these methods is greatly affected by the complex structure of the shell and the internal equipment, the detection sensitivity is low, and the PD characterization data is not comprehensive, which lead to high rate of false and missed reports.

Considering the optical phenomenon of PD, the online monitoring of GIS insulation can be realized by UV photon detection. The optical detection methods are not interfered by the electromagnetic signals which are investigated on the optical detection method of PD by researchers. In respect of fault detection mechanism, in literatures [1-3], GIS simulation models are established, different types of discharges and optical propagation modes are studied, and the attenuation law of optical flux in different structures is obtained. In literatures [4-7], the UV characteristics of long gap discharge are studied by UV detection method, and the nanosecond characteristics of optical pulse are obtained. In respect of detection equipment, photomultiplier tube (PMT) and UV imager are widely used in discharge detection [8-10]. Based on the theory of secondary electron emission, PMT is widely used to convert photons of specific wavelength into available current signal, which takes the advantages of low dark current, low cost and high sensitivity [11-14]. However, there is no obvious regression between the amplitude of the UV intensity and PD, which makes UV method is not widely used for quantitative sensing in GIS.

In this paper, the single photon sensing system based on PMT is built. The UV characteristics of PD are obtained by the real GIS protrusion discharge model. The patterns of UV pulse signal variation in different discharge stages are studied. Combined with the pulse current method, the UV sensing method for quantitative measurement of PD is proposed.

\section{Principle of single photon sensing for protrusion discharge}

\subsection{Principle of $U V$ photon radiation and absorption in GIS}

With the applied high voltage, protrusion discharge is incepted by the extremely uneven field on the surface of gas and solid dielectric. UV photons of different wavelengths $(200 \mathrm{~nm} \sim 400 \mathrm{~nm})$ are produced by intense ionization. The diffusion, drift and recombination of charged particles caused by discharge will further intensify the photon radiation during the progress of the inception of discharge to breakdown.

\footnotetext{
${ }^{1}$ China Electric Power Research Institute, Beijing, 100192, ${ }^{2}$ State Grid Corporation of China, Beijing, 100031, dufei@epri.sgcc.com.cn
} 
With the increasing of the discharge intensity, the high-energy charged particles are accelerated further in the electric field, and oxygen atoms are dissociated on the surface of the insulator, ozone is produced in the three body collision, meanwhile, decomposed product of SF6 gas such as SF5, SF4 and fluorine ion is formed, and UV photons are absorbed [15]. The photon radiation in decomposed product of insulating gas is shown in Fig. 1.

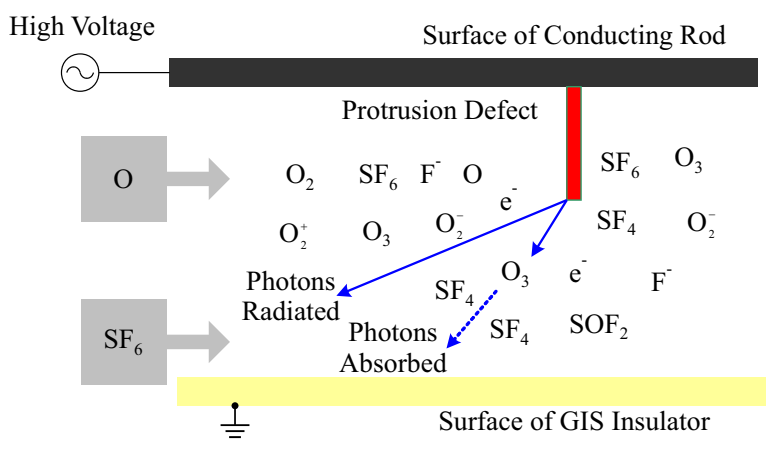

Fig. 1. Photon radiation in GIS

In the discharge area, oxygen atoms, ozone molecules, oxygen molecules and decomposition products of $\mathrm{SF}_{6}$ collide with each other to further generate absorbing gas

$$
\left\{\begin{array}{l}
\mathrm{O}+\mathrm{O}+\mathrm{M} \rightarrow \mathrm{O}_{2}+\mathrm{M} \\
\mathrm{O}+\mathrm{O}_{3} \rightarrow 2 \mathrm{O}_{2} \\
\mathrm{O}+\mathrm{O}_{2}+\mathrm{M} \rightarrow \mathrm{O}_{3}+\mathrm{M} \\
\mathrm{SF}_{6}+\mathrm{e}^{-}=\mathrm{SF}_{6}^{-} \\
\mathrm{SF}_{6}+\mathrm{e}^{-}=\mathrm{SF}_{5}^{-}+\mathrm{F}^{-}
\end{array}\right.
$$

Where $M$ represents the particle participating in the three-body collision. Because it is difficult to measure the gas composition in the actual discharge process, the gas concentration can not be used as the direct basis for calculating the absorption of UV photons.

The absorption cross section of gas $\sigma(\lambda)$ reflects the projection area of each gas molecule in the beam, namely, the absorption ability of gas to photons. According to the Beer-Lambert law, the absorption rate of gas for UV photons is exponentially related to the absorption cross section, as shown in equation (2).

$$
\varepsilon=1-e^{-\sigma(\lambda) n l}
$$

where $n$ represents the number of gas molecules per unit volume, $l$ represents optical path.

\subsection{Characterization parameters of optical and electri- cal signals of protrusion discharge}

Parameters such as apparent discharge capacity, discharge repetition rate and phase resolved partial discharge (PRPD) pattern are mature to characterize the severity of discharge. Similarly, for the optical signal, the parameters such as the amplitude of the optical pulse, the phase distribution of the optical pulse, and the number of photons radiated per unit time can be obtained by the UV detection system, and can be used to characterize the severity of the discharge $[9,10]$. According to equation (1) and (2), the UV photons radiated by the discharge arrive at the UV detector only after being absorbed by the gas, and the absorption rate is related to the wavelength of the photons. However, the gas components in the actual discharge area are difficult to obtain, therefore the severity of the discharge cannot be directly evaluated according to the number of photons. On the other hand, the wavelength of photon is difficult to measure, and the photoelectric conversion efficiency of the UV detector is related to the photon wavelength, therefore the amplitude of the optical pulse can not directly represent the severity of the discharge as well.

Considering the electrical statistical characteristics of the protrusion discharge and the processing method of UV signal, firstly, the discharge area is calibrated by a high sensitivity UV imager to obtain the relative area of the UV spot, and then the gas absorption cross section is calculated to obtain the absorption characteristics of UV photons. For the fixed structure observation window of GIS gas chamber, the facula area is greatly affected by the observation angle and optical path, so the relative facula area under the fixed angle is used to calculate the relative volume change of the discharge area

$$
\bar{S}_{i}=\frac{S_{i}}{S_{1}} \propto\left(\frac{V_{i}}{V_{1}}\right)^{2 / 3}=\left(\frac{n_{i}}{n_{1}}\right)^{2 / 3}
$$

where, $\bar{S}_{i}, S_{i}, V_{i}$ and $n_{i}$ represent the relative facula area under the $i$-th voltage level, the observed results of the UV imager, the discharge area volume and the molecular number respectively. Combined with the high sensitivity single photon sensing system, the regression characteristics of the number of discharge UV pulse signals and absorption characteristics are established, and the regression characteristics of the number of photons and discharge area are calculated, and the periodic change rules of discharge with different severity are obtained. Meanwhile, the discharge capacity is calculated by electrical signal, and the UV sensing system is calibrated to realize the quantitative measurement in different stages of discharge development.

\subsection{Single photon sensing system}

The single UV photon sensing system is composed of photodetector, amplifier, acquisition module and optical signal processing module. The system structure and installation method are shown in Fig. 2(a). The defect model is set on the conductor next to the center of the basin insulator. Photons first arrive at the photodetector (PDM 9107-Q, ET enterprises, UK) through the incident window, and then are received by the photocathode. After 13 stages of amplification, the photoelectric signal is converted into pulse voltage signal, which is collected by acquisition card (ADQ412. 2 GS/s/ch, Sweden), and then 


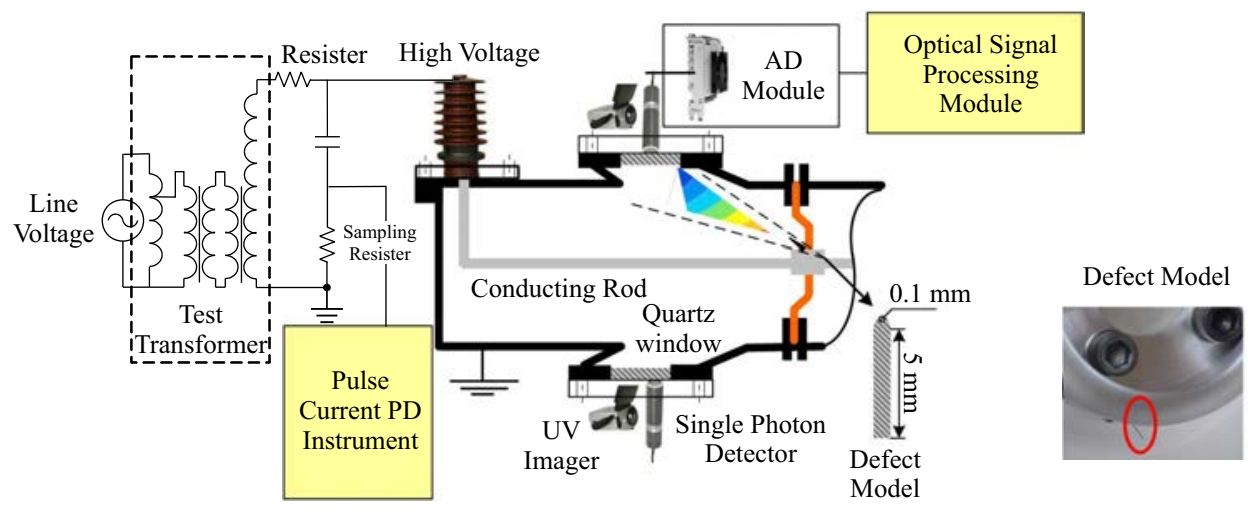

Fig. 2. Test platform for single photon perception of discharge: (a) - schematic diagram of sensing system and test platform, and (b) real test platform
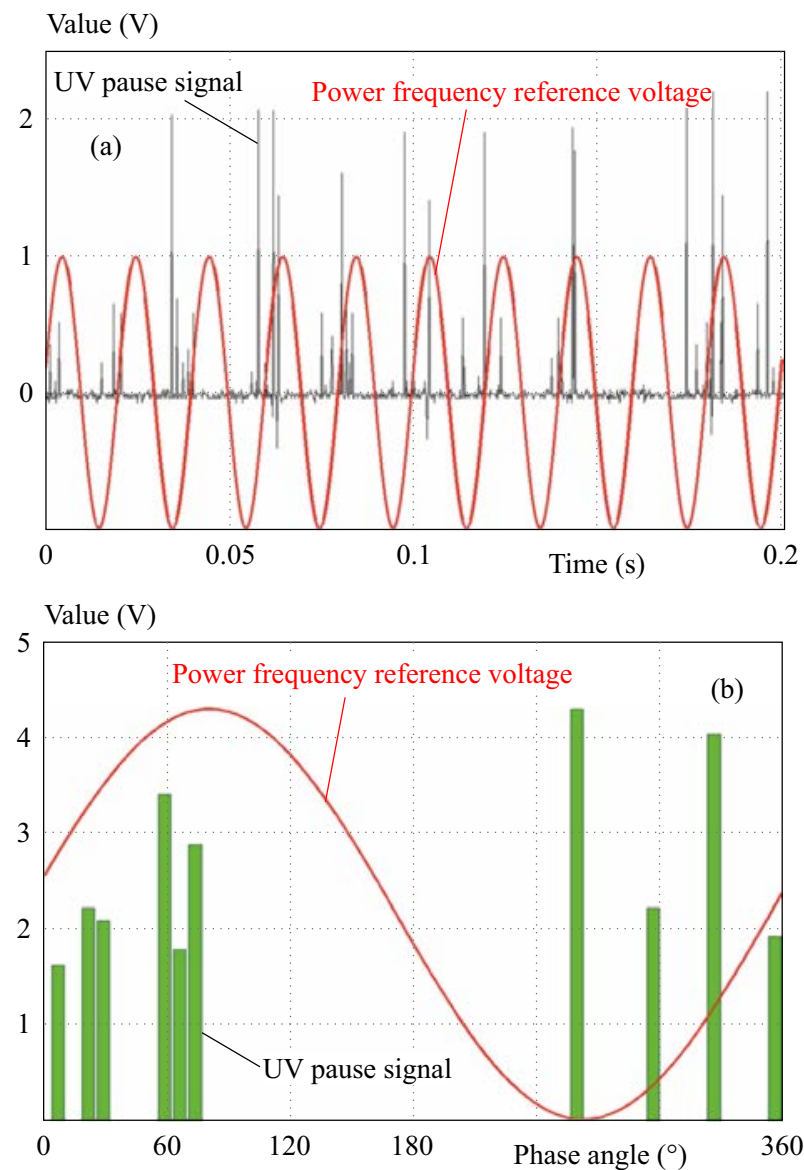

Fig. 3. Test results of optical and electrical test methods at $26 \mathrm{kV}$ : (a) - waveform of UV pulse in time domain. and (b) - pulse current pattern

processed and analyzed by optical signal processing module.

Firstly, the sensing system is tested in the dark room with UV light source. The number of dark current pulses in unit time of the system is obtained by one-hour pulse counting test, namely the noise of the system is calibrated. The system performance parameters with UV source $(240 \mathrm{~nm} 300 \mathrm{~nm})$ are shown in Tab. 1.
Table 1. Performance parameter of a single photon perception system

\begin{tabular}{lcc}
\hline Performance parameter & Value & Unit \\
\hline Spectral range & $260 \approx 280$ & $\mathrm{~nm}$ \\
Pulse rise time & 1.2 & $\mathrm{~ns}$ \\
Pulse fall time & 1.2 & $\mathrm{~ns}$ \\
Pulse pair resolution & 25 & $\mathrm{~ns}$ \\
Dead Time & 25 & $\mathrm{~ns}$ \\
Dark current frequency & 10 & $\mathrm{~Hz}$ \\
\hline
\end{tabular}

\section{Single photon sensing tests for protrusion discharge in GIS}

\subsection{Test platform}

The protrusion discharge test platform is composed of voltage regulation system, protrusion discharge model, pulse current detection system, UV imager and single photon sensing system, as shown in Fig. 2(a). The actual test platform is shown in Figure 2(b). Based on the platform, the UV imager can qualitatively detect whether the discharge occurs, and calculates the relative area of the discharge area

$$
S_{i}=\frac{r_{i}^{2}}{r_{0}^{2}},
$$

where, $s_{i}$ represents the relative area with $i$-th voltage level, $r_{0}$ represents the spot radius of UV image with the partial discharge inception voltage, $r_{i}^{2}$ is the sum of squares of spot radius with $i$-th voltage level. Then the regression relationship between the relative area and the photon number obtained by sensing system can be studied.

\subsection{Test process}

In order to inspire the protrusion discharge of different quantities, stimulate insulation defects of different deterioration level and photon radiation level, step method 
is used. The sensing system and pulse current PD sensor obtain weak PD signals when the applied voltage is $26 \mathrm{kV}$, as shown in Fig. 3. After 10 minutes, the applied voltage is raised to $30 \mathrm{kV}$, and stable $\mathrm{PD}$ signals are obtained by the two test systems. The applied step voltage is chosen as $5 \mathrm{kV}$, and is maintained for 30 mins in each voltage level, and the basin insulator flashover happens at $75 \mathrm{k} \mathrm{V}$. The observation results of the UV imager are used to qualitatively calibrate the UV photons distribution, as shown in Fig. 4. The discharge times in positive half cycle $N^{+}$, discharge times in negative half cycle $N$ -, discharge times in whole cycle $N_{\text {total }}$, relative area of facula $S$, discharge quantity $Q$ at each voltage level are shown in Tab. 2.
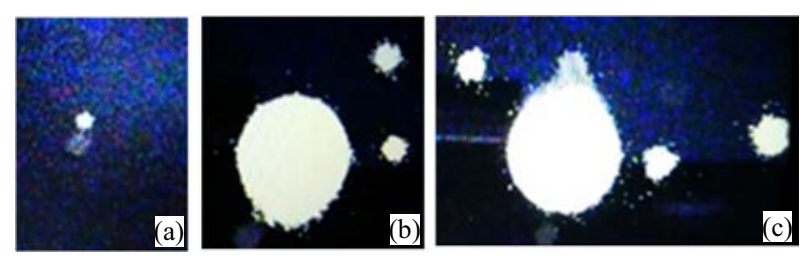

Fig. 4. Observation results of UV imager

Table 2. Results of optical and electrical tests at different voltage

\begin{tabular}{lccccc}
\hline Voltage $(\mathrm{kV})$ & $N^{+}$ & $N-$ & $N_{\text {total }}$ & $S$ & $Q(\mathrm{pC})$ \\
\hline 26 & 5 & 13 & 18 & 1 & 18.2 \\
30 & 9 & 11 & 20 & 4.5 & 20.0 \\
35 & 7 & 11 & 18 & 2.1 & 18.8 \\
40 & 9 & 15 & 24 & 8.2 & 23.5 \\
45 & 6 & 18 & 24 & 8 & 23.6 \\
50 & 4 & 56 & 60 & 21 & 73.5 \\
55 & 46 & 99 & 145 & 71.9 & 123.4 \\
60 & 73 & 135 & 208 & 92.3 & 143.8 \\
65 & 195 & 226 & 421 & 128 & 183.6 \\
70 & 35 & 127 & 162 & 148.5 & 204.4 \\
75 & 114 & 196 & 310 & 255 & 369.2 \\
\hline
\end{tabular}

According to Table 2, relationships between photon number and discharge quantity are analyzed, the relating

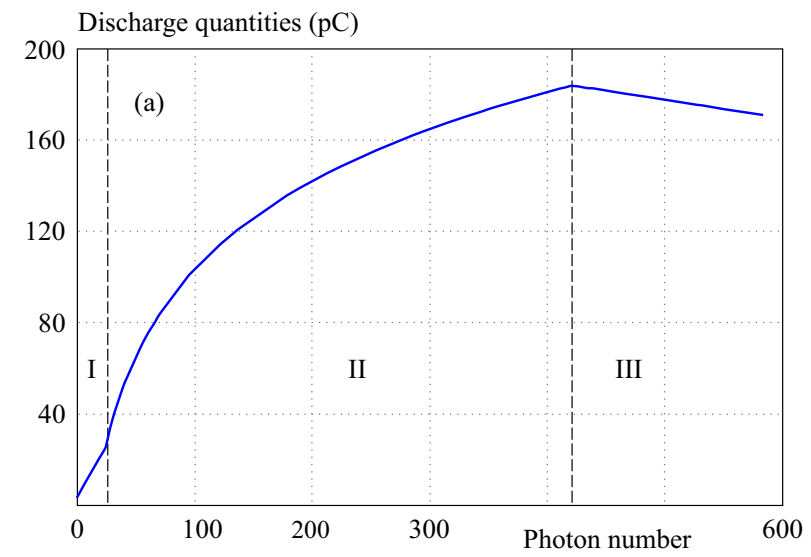

law of discharge number and UV Relative area of facula is shown in Fig. 5.

\subsection{Relationships between photon number and PD development}

The results indicate that, from $26 \mathrm{kV}$ to $65 \mathrm{kV}$, the discharge times increase exponentially with the applied voltage, and the relative area of facula obtained by UV imager is also increase in this stage. While the applied voltage is $70 \mathrm{kV}$, the number of photons obtained by the sensing system decreases, which does not meet the change pattern of previous stage.

Considering that when negative half cycle voltage is applied, the gas molecules are ionized under the high electric field strength or strong rays, such as oxygen anions. The oxygen and fluorine ions move far away from the electrode, but due to the large diameter of the oxygen ions, the mobility of ion in the electric field is lower, then the ions move only a short distance in a half-cycle. At the same time, the negative ions further enhance the electric field on the electrode surface, which increases the radiation intensity of UV photons as well [15-16].

Considering when positive half cycle voltage is applied, the oxygen anions near the electrode move towards the electrode. As the concentration of oxygen anions is higher and higher towards to the electrode, the oxygen anions near the electrode tip are finally stripped of electrons and recombined into ozone [17-19]. Therefore, the concentration of ozone near the electrode is relatively higher. The UV photons excited by the ionization of gas near the electrode are absorbed by ozone, which lead to less pulse signals obtained by the sensing system. In addition, this phenomenon explains how the UV pulse signal is generated in the rising stage of positive half cycle, namely, the applied voltage gradually increases during the positive half cycle, the negative oxygen ion will not completely lose electrons and recombine to ozone, the concentration of ozone near the electrode is relatively lower, and then the UV photons produced by the electrode can be radiated through the discharge reign to the sensing system by field emission.

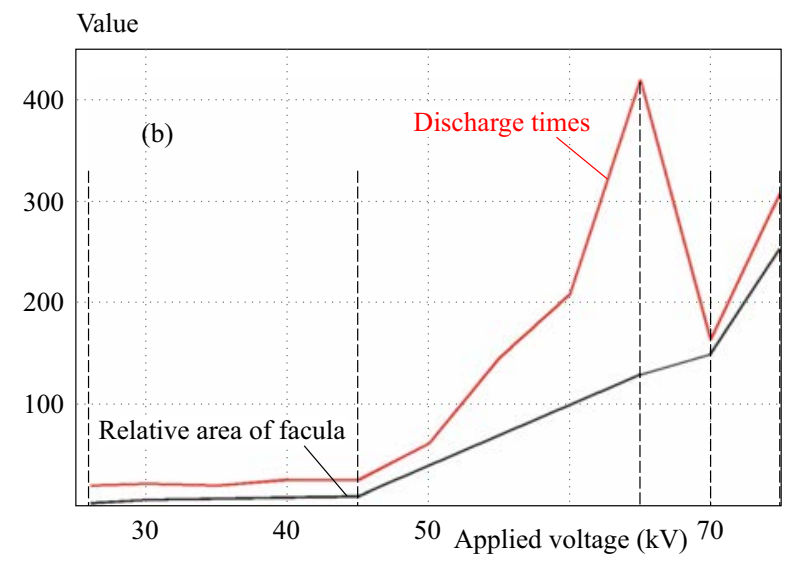

Fig. 5. Trends of radiation photons at different voltage: (a) - trend of total discharge times and discharge quantities. and (b) - trend of total discharge times and relative area of facula 


\section{Phased quantitative analysis for optical signals of discharge}

\subsection{Initial stage of discharge}

Figure 5(b) indicates that the development of discharge can be divided into four stages. The first stage is the initial stage of discharge, whose applied voltage range is $26 \mathrm{kV}$ to $45 \mathrm{kV}$. In this stage, the relative area of facula is smaller, increasing more slowly and the discharge times are smaller. The approximate relationships between the relative area of facula $S_{1}$ and the discharge times $N_{1}$ and the discharge quantities $P_{1}$ are

$$
\left\{\begin{array}{l}
N_{1}=0.857 S_{1}+17 \\
P_{1}=0.9 N_{1}+2
\end{array} .\right.
$$

Equation (5) further indicates that, due to the low amplitude of the electric field, stable ozone can not be formed, and the solar blind UV photons can always be radiated from the discharge position to the PMT, then the discharge detection is easily realized. The solar blind UV photons are inspired in two ways: (1) the field emission of the metal electrode; (2) the electron level transition during the gas ionization and recombination.

\subsection{Development stage of discharge}

The second stage is the development stage of discharge, whose applied voltage range is $45 \mathrm{kV}$ to $65 \mathrm{kV}$. In this stage, the insulation gradually deteriorates, and the relative area of facula is linear with the applied voltage, while the total discharge times obey the law of exponential increasing. The relationships between the discharge times N2 and the relative area of facula $\mathrm{S} 2$, the discharge quantities $P_{2}$ are

$$
\left\{\begin{array}{l}
N_{2}=41.33 \exp \left(0.0177 S_{2}\right) \\
P_{2}=52+56.5 \ln \frac{N_{2}}{41}
\end{array},\right.
$$

indicating that the UV photons radiated by the metal electrode are partially absorbed by ozone during the positive half cycle, and the field emission of the metal electrode is not the main reason of the UV photons radiation, instead, the avalanche of electrons in the discharge area, new photons by electron transition are the main reasons which lead to the generation of new photons.

\subsection{Stable stage of discharge}

The third stage is stable stage of discharge, whose applied voltage range is $65 \mathrm{kV}$ to $70 \mathrm{kV}$. In this stage, the patterns of UV photons emission are relatively stable. The relative area of facula increases slightly. The number of UV photons radiated during the positive half cycle decrease obviously, which indicates that the ozone concentration was higher than the previous stage, large number of UV photons produced in the positive half cycle are absorbed as well. In the negative half cycle, because the concentration of fluoride and ozone is still high, ozone molecules and fluoride such as $\mathrm{SF}_{4}$ can not be electrolyzed to oxygen anion and fluorine ion rapidly, which leads to a large reduction of UV photons. Due to the high energy of charged particles in the discharge area, photons are radiated without absorbed in the areas with lower ozone and fluoride concentration

$$
\left\{\begin{array}{l}
N_{3}=-12.639 S_{3}+2038.818 \\
P_{3}=-0.08 N_{3}+217.33
\end{array}\right.
$$

The last equation indicates that, the radiation ability of the charged particles in the outer layer of the discharge area is much higher than the field emission of the first stage electrode, but negative radiation ability is presented in the inner layer of the discharge area. The phenomena and equation further illustrate that, with the increase of the applied voltage, the ozone and fluoride concentration increase, and the ability to absorb photons is also stronger, which is not conducive to UV detection.

\subsection{Stage of hybrid discharge}

The fourth stage is the stage of hybrid discharge, whose applied voltage range is $70 \mathrm{kV}$ to $75 \mathrm{kV}$. In this stage, not only discharge occurs near the tip of the electrode, but also at other positions of the electrode. The first, second and third stages of discharge happens at the same time. Therefore, it can be used to verify the rationality of the analysis of the first three stages. The relative area of facula is 169 at the tip electrode position, 9 at other metal positions and 77 in the air, namely, $S_{1}=9$, $S_{2}=86, S_{3}=169$. According to the equations (5) (7), the number of discharge times is 311 . According to the real test, the number of discharge times is 310 , the error is 1 , which is in the range of dark current error. The calculated value of hybrid discharge is $388.3 \mathrm{pc}$, and the error is $5.2 \%$, which proves that the above analysis of three stages are reasonable.

\section{Conclusion}

Based on the single UV photon sensing system, the evolution of UV photoelectric phenomenon induced by protrusion discharge in GIS is studied, and the corresponding relationship between UV and electric signals in different stages of discharges development are obtained, which can be used to realize high sensitivity discharge monitoring, quantitative monitoring and development process judgment of GIS internal discharge. The conclusions are as follows:

(1) The sensitivity of single UV photon sensing system is equal to pulse current method. A calibration method of single photon sensing system based on relative area of facula is proposed. The discharge process is divided quantitatively, and the regression relationships between $\mathrm{PD}$ and number of photons in each process are obtained quantitatively.

(2) According to the regression relation of photons' number, relative area of facula and discharge quantity, the 
protrusion discharge of GIS basin insulator can be divided into four stages: initial stage of discharge, development stage of discharge, stable stage of discharge and stage of hybrid discharge.

(3) With the increase of applied voltage, the number of UV photons gained by sensing system is not always positively related to the insulation degradation, but is related to the diffusion, migration and recombination of charged particles.

\section{Acknowledgments}

This project is supported by Research and application of optical and electrical sensing technology with high accuracy for partial discharge in GIS, the Technical Project of State Grid Corporation of China(5600-202021159A-0$0-00)$.

\section{REFERENCES}

[1] X. Han, Z. Liu, L. Zhang, et al "Simulation for Propagation Characteristics of Optical Signals Excited by Partial Discharge GIS", Journal of Xian Jiaotong University, vol. 52, no. 6, pp. 128-134, 2018.

[2] C. Zhuang, R. Zeng, B. Hang, et al "A WENO scheme for simulating streamer discharge with photoionizations", IEEE Transactions on Magnetics, vol. 50, no. 2, pp. 7007904, 2014.

[3] C. Zhuang and R. Zeng, "A positivity-preserving scheme for the simulation of streamer discharges non-attaching and attaching gases", Communications Computational Physics, vol. 15, no. 1, pp. 153-178, 2014.

[4] W. Chen, R. Zeng, and H. He "Research progress of long air gap discharge", High Voltage Engineering, 2013, 39(6):1281-1295.

[5] F. Rizk, "Critical switching impulse strength of long air gaps: modelling of air density effects", IEEE Transactions on Power Delivery, vol. 7, no. 3, pp. 1507-1515, 1992.

[6] E. Bazelyan, Y. Raizer, and N. Aleksandrov, "The effect of reduced air density on streamer-to-leader transition and on properties of long positive leader", Journal Physics D: Applied Physics, vol. 40, no. 14, pp. 4133-4144, 2007.

[7] Z. Wang, C. Zhuang, Y. Zhang, et al "Measurement of Streamer Propagation Velocity Based on Photomultiplier", High Voltage, vol. 44, no. 3, pp. 920-925, 2018.

[8] L. Dai, "Application of UV imaging technology live detection of high voltage equipment", Autormation of Electric Power Systems, vol. 27, no. 20, pp. 97-98, 2003.

[9] Q. Li, T. Chen, J. Wang, et al "Application of UV Pulse Method on the UHV Discharge Detection", High Voltage, vol. 32, no. 12, pp. 26-29, 2007.

[10] S. Wang, B. Mei, Z. Ye, et al "Ultraviolet Imaging Detection Technology and Application Corona Discharge Detection of Electrical Equipment", High Voltage Apparatus, vol. 47, no. 11, pp. 92-97, 2011.

[11] X. Dang, "Preparation and Properties of Solar-blind Photomultiplier Tube", Opto-Electronic Engineering, vol. 46, no. 6, pp. 53-58, 2019.

[12] W. Zhao, "Developments technology of Photomultipliers", Optoelectronic Technology, vol. 31, no.3, pp. 145-148, 2011.

[13] W. Zhao, D. Liu, J. Ding, et al "Developed and Time Characteristic Measure Researched of the Ultra-fast Photoelectric Multiplier", Optoelectronic Technology, vol. 28, no. 4, pp. 267-269, 2008.
14] N. Atanov, V. Baranov, J. Budagov, et al "Quality Assurance on Un-Doped CsI Crystals for the Mu2e Experiment", IEEE Transactions on Nuclear Science, vol. 65, no. 2, pp. 752-757, 2018.

[15] M. Li, B. Peng, L. Wei, et al "Numerical simulation for the effects of pulse power parameters on ozone generation by pulsed dielectric barrier discharge", High Voltage Engineering, vol. 42, no. 8 , pp. 2659-2667, 2016.

[16] M. Malik and K. Schoenbach, "Nitric Oxide Conversion and Ozone Synthesis a Shielded Sliding Discharge Reactor with Positive and Negative Streamers", Plasma Chemistry \& Plasma Processing, vol. 34, no. 1, pp. 93-109, 2014.

[17] Z. Liu, Y. Wu, and N. Wang, "Experimental Study on Ozone Synthesis Dielectric Barrier Discharge Triggered by Bipolar Narrow Pulse", High Voltage, vol. 27, no. 2, pp. 28-29, 2001.

18] S. Kyung, J. Park, J. Lee, et al "Study of selective amorphous silicon etching to silicon nitride using a pin-to-plate dielectric barrier discharge atmospheric pressure", Applied Physics Letters, vol. 91, no. 9, pp. 091504.1-091504.32007, 2007.

[19] M. Chang and S. Wu, "Experimental Study on Ozone Synthesis via Dielectric Barrier Discharges", Ozone: Science \& Engineering, vol. 19, no. 3, pp. 241-254, 1997.

Received 6 July 2020

Fei Du was born in Beijing, China, in 1992. He received the MS in electric and engineering from Xi'an Jiao Tong University (Xi'an) in 2017, respectively. Currently, he is an Engineer with China Electric Power Research Institute, Beijing, China. His research interests are condition monitoring of power apparatus.

Wenzhi Chang was born in Gansu, China, in 1983. He received the $\mathrm{MS}$ and $\mathrm{PhD}$ degrees in electrical engineering from North China Electric Power University (Beijing) in 2009 and 2013, respectively. Currently, he is a Senior Engineer with China Electric Power Research Institute, Beijing, China. His research interests are condition monitoring of power apparatus.

Jiangang Bi was born in Inner Mongolia, China, in 1977. He received the MS in control science and engineering from Xi'an Jiao Tong University (Xi'an) in 2003, respectively. Currently, he is a Professor Level Senior Engineer with China Electric Power Research Institute, Beijing, China. His research interests are condition monitoring of power apparatus.

Shuai Yuan was born in Inner Mongolia, China, in 1980. He received the PhD degrees in Harbin Institute of Technology in 2009. Currently, he is a Senior Engineer with China Electric Power Research Institute, Beijing, China. His research interests are condition monitoring of power apparatus.

Xinghui Zhang was born in Beijing, China, in 1982. He received the MS degree in Southeast university in 2014. Currently, he is a Senior Engineer with State Grid Corporation of China. His research interests are operation and maintenance of power equipment.

Yanpeng Gong was born in Hebei, China, in 1983. He received the MS degree in electrical engineering from China Electric Power Research Institute in 2009. Currently, he is a Senior Engineer with China Electric Power Research Institute, Beijing, China. His research interests are condition monitoring of power apparatus. 